\title{
Foreword to the special issue on the 2017 Static Analysis Symposium
}

\author{
Francesco Ranzato ${ }^{1}$ (D) \\ Published online: 26 August 2019 \\ (c) Springer Science+Business Media, LLC, part of Springer Nature 2019
}

\begin{abstract}
This volume of Formal Methods in System Design (FMSD) features extended and revised versions of a selection of papers presented at the Static Analysis Symposium (SAS) held on 2017 in New York, NY. The series of Static Analysis Symposia serves as a primary venue for the presentation of theoretical, practical, and applicational advances in the area of static analysis of programs and systems. After the conference, the Program Committee selected the best papers of SAS 2017 and invited their authors to submit an extended version. This special issue of FMSD contains the four high-quality articles that were accepted after a careful reviewing process.
\end{abstract}

\section{Background}

This special issue of Formal Methods in System Design (FMSD) features extended and revised versions of a selection of papers presented at the 24th Static Analysis Symposium (SAS), held on August 30th-September 1st, 2017, in New York, NY, hosted by the Courant Institute of Mathematical Studies of the New York University, and organized by the local chair Patrick Cousot.

Static Analysis is widely recognized as a range of fundamental principles, techniques and tools for program verification, bug detection, compiler optimization, program transformation, program synthesis, program understanding and software maintenance. The community of researchers, practitioners, teachers and professional users of static analysis is growing both in academia and industry and novel application areas are quickly emerging, notably static analysis of machine learning algorithms and of blockchain smart contracts. The series of Static Analysis Symposia serves as a major venue for the presentation of theoretical, practical, and applicational advances in the area. This volume pursues this goal and allows to selected authors of SAS 2017 to present additional results, extensions, benchmarks and proofs.

SAS 2017 attracted 50 submissions, each of which was reviewed by at least three Program Committee members. Following a two months reviewing period, the Program Committee accepted 22 papers for publication and presentation at the conference in New York. Soon after the conference, the Program Committee selected the best papers and invited their authors

Francesco Ranzato

francesco.ranzato@unipd.it

1 Dipartimento di Matematica, University of Padova, Padua, Italy 
to submit an extended and revised version to this special issue of FMSD. Among them, four articles were eventually accepted. After a thorough review process that required two to three rounds of revisions for each paper, we are delighted to publish these final extended versions.

We would like to thank all the authors for their excellent work, all the reviewers who contributed to improve the quality of the papers, Daniel Kroening, who acts as FMSD Editor in Chief, and the Springer Support team who made the preparation and publication of this volume possible.

\section{Selected papers}

In the first paper "Abstract Semantic Diffing of Evolving Concurrent Programs", Boujjani et al. [1] put forward a novel methodology for computing the semantic differences between two closely related concurrent programs (that is, subsequent versions of a program). This framework relies on a semantics based on program traces, modelling program action orders and read/write orders. This trace semantics is approximated by a number of parameterized abstractions which are then compared through partial order relations and allow to define succinct representations of the semantic difference between two concurrent programs.

In the second contribution "Template Polyhedra and Bilinear Optimization", Gronski et al. [2] introduce a new technique for computing abstract inductive invariants of transition systems, where an invariant is represented by a polyhedron which satisfies some parameterized template. This class of template domains generalize well-known numerical abstract domains such intervals, octagons and linear/quadratic templates. In particular, the authors consider the problem of checking the existence of polyhedral invariants for a given set of templates specified as a satisfiability problem over bilinear constraints used in classic bilinear optimization problems.

In the third article "Quantitative Static Analysis of Communication Protocols using Abstract Markov Chains", Ouadjaout and Miné [3] present a static analysis for inferring symbolic quantitative properties of probabilistic programs, in particular focussed on analysing communication protocols. This analysis is designed within the abstract interpretation framework and relies on a new abstract Markov chains domain which provides an over-approximation of the behaviour of probabilistic programs on discrete distributions with limited nondeterministic choices. The analysis exploits a parametric Fourier-Motzkin elimination algorithm which yields symbolic bounds on the probabilities of program properties.

In the last paper "A New Abstraction Framework for Affine Transformers", Sharma and Reps [4] present an abstract interpretation-based static analysis of numerical properties of programs. This approach makes use of a program semantics given by sets of affine transformers over bit-vectors, which are then abstracted using existing numeric abstract domains. This provides an extension of a previous work by Müller-Olm and Seidl, which was limited to abstractions consisting of affine equalities, while the proposed framework can be instantiated with arbitrary abstract domains.

\section{References}

1. Bouajjani A, Enea C, Lahiri SK (2018) Abstract semantic diffing of evolving concurrent programs. Formal Methods Syst Design. https://doi.org/10.1007/s10703-018-0322-2

2. Gronski J, Ben Sassi MA, Becker S, Sankaranarayanan S (2018) Template polyhedra and bilinear optimization. Formal Methods Syst Design. https://doi.org/10.1007/s10703-018-0323-1 
3. Ouadjaout A, Miné A (2019) Quantitative static analysis of communication protocols using abstract Markov chains. Formal Methods Syst Design. https://doi.org/10.1007/s10703-019-00331-2

4. Sharma T, Reps T (2018) A new abstraction framework for affine transformers. Formal Methods Syst Design. https://doi.org/10.1007/s10703-018-0325-z

Publisher's Note Springer Nature remains neutral with regard to jurisdictional claims in published maps and institutional affiliations. 